\title{
This Story Needs to Be Told: Formative Research on Using Animated Storytelling to Address Prospective Minority Clinical Trials Participants' Need for Comprehensive Knowledge
}

\author{
Stephanie M. Younker ${ }^{1}$, Katherine E. Ridley-Merriweather ${ }^{2}$ \\ ${ }^{1}$ Indiana University School of Medicine, ${ }^{2}$ Susan G. Komen Tissue Bank at the IU \\ Simon Cancer Center
}

Background: The Komen Tissue Bank (KTB), a clinical trial that collects women's healthy breast tissue for use as research study controls, currently stores samples demonstrating a noted paucity of minority specimens. Studies have shown minority groups' participation in clinical trials is dependent upon the groups' knowledge about the associated research. This study's purpose is to conduct formative research on animated storytelling's effectiveness as a communication tool to address minority groups' expressed need for knowledge and encourage participation in cancer clinical trials.

Methods: I re-coded data previously gathered from a KTB study to evaluate the perceptions of Latinas $(n=14)$ and Asian Americans $(n=17)$ toward breast tissue donation. Extensive review of the current literature yielded data concerning African Americans' outlooks toward clinical trial participation, and also illustrated the efficacy of using narratives and animation as communication and education tools.

Results: The literature review clearly illuminated the importance of storytelling in minority cultures and suggested the success of using narratives as communication tools. We anticipate our findings could be influenced by a) minorities' lack of, and need for, comprehensive knowledge, b) previously obscured participation barriers, and c) animated storytelling as an effective delivery method.

Projected Conclusion and Potential Impact: Using an animated storytelling communication tool may successfully increase knowledge about, and comfort with, participation in a cancer clinical trial. The next phase, an animated video production, will tell the comprehensive story of healthy breast tissue donation. The use of this tool could help increase minority participation in cancer prevention and cancer clinical trials. 